\title{
Hydrogel versus Bare Platinum Coils in Patients with Large or Recurrent Aneurysms Prone to Recurrence after Endovascular Treatment: A Randomized Controlled Trial
}

\author{
(D). Raymond, DR. Klink, (D) M. Chagnon, S.L. Barnwell, (D)A.J. Evans, DJ. Mocco, (D)B.H. Hoh, (D)A.S. Turk, (D) R.D. Turner, DH. Desal,

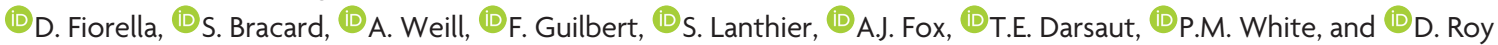

A

\begin{abstract}
BACKGROUND AND PURPOSE: Some patients are at high risk of aneurysm recurrence after endovascular treatment: patients with large aneurysms (Patients Prone to Recurrence After Endovascular Treatment PRET-1) or with aneurysms that have previously recurred after coiling (PRET-2). We aimed to establish whether the use of hydrogel coils improved efficacy outcomes compared with bare platinum coils.

MATERIALS AND METHODS: PRET was an investigator-led, pragmatic, multicenter, parallel, randomized (1:1) trial. Randomized allocation was performed separately for patients in PRET-1 and PRET-2, by using a Web-based platform ensuring concealed allocation. The primary outcome was a composite of a residual/recurrent aneurysm, adjudicated by a blinded core laboratory, or retreatment, intracranial bleeding, or mass effect during the 18-month follow-up. Secondary outcomes included adverse events, mortality, and morbidity (mRS $>$ 2). The hypothesis was that hydrogel would decrease the primary outcome from $50 \%$ to $30 \%$ at 18 months, necessitating 125 patients per group (500 for PRET-1 and PRET-2).

RESULTS: The trial was stopped once 250 patients in PRET-1 and 197 in PRET-2 had been recruited because of slow accrual. A poor primary outcome occurred in $44.4 \%(95 \% \mathrm{Cl}, 35.5 \%-53.2 \%)$ of those in PRET-1 allocated to platinum compared with $52.5 \%(95 \% \mathrm{Cl}, 43.4 \%-61.6 \%)$ of patients allocated to hydrogel (OR, 1.387; 95\% Cl, 0.838-2.295; $P=.20)$ and in $49.0 \%(95 \% \mathrm{Cl}, 38.8 \%-59.1 \%)$ in PRET-2 allocated to platinum compared with $42.1 \%(95 \% \mathrm{Cl}, 32.0 \%-52.2 \%)$ allocated to hydrogel (OR, 0.959; 95\% Cl, 0.428-1.342; $P=.34)$. Adverse events and morbidity were similar. There were $3.6 \%$ deaths $(1.4 \%$ platinum, $5.9 \%$ hydrogel; $P=.011)$.
\end{abstract}

CONCLUSIONS: Coiling of large and recurrent aneurysms is safe but often poorly effective according to angiographic results. Hydrogel coiling was not shown to be better than platinum.

ABBREVIATION: PRET $=$ Patients Prone to Recurrence After Endovascular Treatment

$E^{n}$ ndovascular coiling has revolutionized the management of intracranial aneurysms. ${ }^{1,2}$ Coiling has been shown safe and effective in the treatment of ruptured aneurysms compared with

Received November 8, 2016; accepted after revision December 14.

From the Departments of Radiology (J.R., A.W., F.G., D.R.) and Neurosciences (S.L.) Centre Hospitalier de l'Université de Montreal, Montreal, Quebec, Canada; Laboratory of Interventional Neuroradiology (R.K.), Research Centre of the Centre Hospitalier de l'Université de Montreal, Quebec, Canada; Department of Mathematics and Statistics (M.C.), Université de Montréal, Montreal, Quebec, Canada; Department of Neurological Surgery (S.L.B.), Oregon Health and Science University, Portland, Oregon; Department of Radiology and Medical Imaging (A.J.E.), University of Virginia, Charlottesville, Virginia; Department of Neurosurgery (J.M.), Mount Sinai Health System, New York, New York; Department of Neurosurgery (B.H.H.), University of Florida, Gainesville, Florida; Department of Neurosurgery (A.S.T., R.D.T.), Medical University of South Carolina, Charleston, South Carolina; Service de Neuroradiologie Diagnostique et Interventionnelle (H.D.), Centre Hospitalier Universitaire de Nantes, Nantes, France; Cerebrovascular Center (D.F.), Stony Brook University Medical Center, Stony Brook, New York; Département de Neuroradiologie Diagnostique et Interventionnelle (S.B.), Centre Hospitalier Universitaire de Nancy, Nancy, France; Department of Medical Imaging (A.J.F.), University of Toronto, Toronto, Ontario, Canada; Department of Surgery (T.E.D.), Division of Neurosurgery, University of Alberta Hospital, Edmonton, Alberta, Canada; and Stroke Research Group (P.M.W.), Institute of Neuroscience, Newcastle Upon Tyne, UK. surgical clipping. ${ }^{3}$ Coiling is also frequently used to preventively treat unruptured aneurysms, even though it has never been proved superior to clipping or observation. ${ }^{4,5}$ One drawback of coiling is the occurrence of residual or recurrent aneurysms at follow-up angiography in $20 \%-30 \%$ of patients. ${ }^{6-8}$ The impact of recurrent aneurysms on long-term clinical outcomes remains unclear. They have infrequently been associated with subarachnoid hemorrhage, in the range of $0.1 \%-1 \%$ per year. ${ }^{7,9}$ Nevertheless, concerns for recurrences have a number of clinical consequences,

This work was supported by an unrestricted research grant from MicroVention Terumo.

Please address correspondence to Jean Raymond, MD, Centre Hospitalier de l'Université de Montréal, Notre-Dame Hospital, Department of Radiology, 1560 Sherbrooke East, Pavilion Simard, Suite Z12909, Montreal, Quebec, Canada H2L 4Ml; e-mail: jean.raymond@umontreal.ca

\footnotetext{
- Indicates open access to non-subscribers at www.ajnr.org

Indicates article with supplemental on-line tables.

Evidence-Based Medicine Level 1.

http://dx.doi.org/10.3174/ajnr.A5101
} 
such as routine angiographic surveillance of nearly all patients, retreatment in 5\%-15\%, and, more recently, the emergence and growing use of potentially more effective but also potentially more risky alternatives, such as stent placement or flow diversion. ${ }^{10-13}$

A randomized trial comparing hydrogel and platinum coiling published in 2011 failed to confirm its primary composite endpoint in favor of hydrogel coils but demonstrated improved core laboratory adjudicated angiographic outcomes overall and in prespecified subgroups of medium (5.0-9.0 mm) and ruptured aneurysms. ${ }^{11,14}$ There was no difference in retreatment rates. A recent systematic review of randomized trials concluded with a reduction of residual aneurysms after hydrogel compared with platinum coiling, but results were barely significant. ${ }^{15}$ Recurrent aneurysms after platinum coiling are at high risk of re-recurring when retreated, but these patients were excluded from all published randomized trials. ${ }^{8,14,16-18}$

The Patients Prone to Recurrence After Endovascular Treatment (PRET) study was designed in 2007 to offer an alternative to platinum coiling in patients previously shown to be at high risk of recurrence: patients with large ( $\geq 10 \mathrm{~mm}$ ) or recurrent aneurysms after coiling. The trial protocol was published in 2008. ${ }^{19} \mathrm{We}$ aimed to establish whether the use of hydrogel coils improved efficacy outcomes compared with bare platinum coils, without increasing procedural risks. Similar periprocedural (30 days) outcomes were reported for hydrogel and platinum coiling. ${ }^{20} \mathrm{We}$ now report the primary outcome of the trial: The primary hypothesis was that the use of hydrogel coils would decrease the proportion of residual or recurrent aneurysms from 50\% to 30\% (range from $50 \%-40 \%$ to $30 \%-21 \%$ ) at 18 months compared with bare platinum coils.

\section{MATERIALS AND METHODS Study Design}

PRET was an investigator-led, pragmatic, multicenter, international, randomized (1:1), controlled trial comparing a policy of using hydrogel versus bare platinum coils in the endovascular treatment of intracranial aneurysms. There were 25 participating clinical sites from 6 countries (United States, Canada, United Kingdom, France, Chile, Japan). The ClinicalTrials.gov registration number from the US National Institutes of Health is NCT00626912. All trial sites had local institutional review board approval. All patients (or legal representatives) signed a standardized informed consent form.

During the course of the trial, no change in methods or protocol occurred, but multiple different types of hydrogel coils were being manufactured, approved, and used.

\section{Participants}

Eligible patients, with an intracranial aneurysm requiring endovascular treatment, fell into 1 of 2 groups: PRET-1, with a large aneurysm (longest dimension, $\geq 10 \mathrm{~mm}$, including any thrombosed portion), never treated; PRET-2, with an aneurysm of any size, presenting with a postcoiling recurrence requiring retreatment. There were few selection criteria: The patient was 18 years of age or older; life expectancy was $>2$ years; the aneurysm could be ruptured (World Federation of Neurologic Societies grade
$<$ IV) or unruptured; anatomy was such that endovascular treatment was considered possible with both types of coils; the endovascular operator was satisfied with using either type of coil, but no other type (such as polyglycolic acid/lactide copolymer coils); and the patient or authorized representative had given fully informed consent and had signed the consent form. Patients were not eligible if they met any of the following criteria: the presence of other aneurysms requiring treatment during the same session; the presence of an associated cerebral arteriovenous malformation; the primary intent of the procedure being parent vessel occlusion without simultaneous endovascular coiling of the aneurysm; and any absolute contraindication to endovascular treatment, angiography, or anesthesia.

Trial sites were tertiary centers experienced in the endovascular treatment of aneurysms with both platinum coils (at least 100 patients treated before enrollment) and hydrogel-coated coils (at least 10 patients previously treated).

\section{Interventions}

Standard local endovascular procedures were followed. Any locally approved bare platinum coil with controlled detachment was permitted, as were any assist devices believed necessary by the operator, provided they had local regulatory approval, excluding flow diverters, irrespective of the intended use indicated at randomization. Antiplatelet and anticoagulation regimens were left to the individual operator's judgment, according to the clinical practice at each site. When treatment allocation was to "platinum," types of coils other than bare platinum were forbidden. When treatment allocation was to "hydrogel," any coil of the hydrogel family was allowed but any bare platinum coil could also be used if the operator believed it was in the patient's best interest. Recommendations concerning hydrogel coil use pertaining to type, size, and sequence of introduction were issued but not enforced. No minimum percentage of hydrogel coils was prescribed; the protocol required "the substitution, as far as possible, of platinum by hydrogel coils, the operator always being allowed to use the coils he or she believes [are] appropriate at any time during the procedure." 15 "Successful hydrogel coiling" was predefined as two-thirds total coil lengths being hydrogel coils, to be used for explanatory analyses only. Other technical considerations such as preparing or steaming of hydrogel coils and the type of bare platinum coil were left entirely to the operator's discretion. The goal of the procedure was to occlude the aneurysm as completely as possible, keeping the risks of the procedure as low as possible. ${ }^{15}$

\section{Outcomes}

The primary outcome of the trial was the proportion of patients with a recurrence, defined as the following: 1) major angiographic recurrence of the lesion or the presence of a residual aneurysm at last angiographic follow-up, as determined by the core laboratory, blinded to treatment allocation; 2) retreatment of the same aneurysm by endovascular or surgical means during the 18-month follow-up period; and 3) an intracranial bleeding episode or the occurrence or progression of a mass effect in relation to the treated aneurysm during the follow-up period, as determined by the blinded Adverse Event Committee. Immediate treatment failures and missing follow-up angiographic data because of treat- 
ment or aneurysm-related deaths or poor clinical outcomes $(\mathrm{mRS}>2)$ were treated as primary outcome events.

Secondary outcomes included safety data, mortality and morbidity, and adverse events, defined as an event of any severity being possibly or probably related to the disease or the treatment and happening at any time during the 18-month follow-up. The independent Adverse Event Committee was responsible for the attribution of secondary outcome events. The protocol prespecified that morbidity would be defined per patient, according to the $m R S$ score ( $m R S>2$ ). Adverse event reports and individual case report forms were cross-checked to determine the secondary safety endpoint for each patient. Periprocedure safety endpoints were reported. Delayed safety outcomes were categorized as the following: subarachnoid hemorrhage, progressive mass effect, stroke, transient ischemic events, inflammatory complications potentially related to coils, non-neurologic, retreatment-related, and others.

\section{Number of Patients}

Two hundred fifty subjects (125 in each group) were judged necessary to achieve $80 \%$ power at a 2 -sided .0250 significance level to detect a difference of .20 (between .30 and .50) in primary outcome events between the intervention and control groups for patients in PRET-1 and PRET-2 separately, assuming a 10\% proportion of patients lost during follow-up. ${ }^{19}$

\section{Randomization}

Randomized allocation (1:1) with minimization of risk factors was through the Web-based PRET application package (MedSciNet, Stockholm, Sweden; http://medscinet.com/), ensuring that allocation was concealed before the decision to include a patient. The minimization algorithm computes an imbalance score for each new patient on the basis of patient characteristics (the minimization criteria) and treatment assignments and characteristics of already-enrolled patients, with treatment with the lowest imbalance score being assigned; in addition, the minimization algorithm is dynamic in that it has a built-in random element for assigning patients to the treatment. We used the following minimization criteria: rupture status (yes, no); if the aneurysm was unruptured, planned use of an adjunct device (yes, no). From the moment of randomization, the patient was in the trial and accounted for in the analysis (intention-to-treat). PRET-1 and PRET-2 patient groups were randomized separately.

\section{Masking and Trial Monitoring}

Masking of the treatment teams was not possible. Patients were masked unless they specifically requested otherwise. The Adverse Event Committee and the Data Safety and Monitoring Committee, working independently from the Steering Committee, had access to masked data, but the Data Safety and Monitoring Committees could unmask groups whenever members thought that unmasking was mandatory to protect the safety of participants, though the need for unblinding did not arise during the trial. Monitoring of trial data quality was Web-based and performed by periodic review of data stored in the data base.

\section{Data Collection}

Data capture and management were held independent of the sponsor and funder on the secure servers of MedSciNet, ISO27000 and Statement on Auditing Standards-70 compliant. Details of data collection are given elsewhere. ${ }^{20}$ Briefly, following registration, intervention, and discharge data collection, clinical follow-up data were recorded at 1, 6, and 18 months and angiographic follow-up imaging was performed at 6 and 18 months. Adverse events and additional interventions on the target aneurysm were reported at any time during the trial. When an additional intervention was planned following the 18-month angiographic follow-up, the form allowed the recording of this event (yes/no check box), though some sites also chose to complete an additional intervention form as well. Anonymized angiographic imaging data (catheter or noninvasive, including additional procedures) were sent in batches to the independent core laboratory (lead, P.M. White, Stroke Research Group, Institute of Neuroscience, Newcastle upon Tyne, United Kingdom) for adjudication of location, dimension, and occlusion state of the aneurysm. The core laboratory was masked to treatment allocation and treatment received. Assessment of the occlusion state was according to the revised 3-point Montreal scale; evolution (stable, better, worse) and occurrence of a major recurrence, defined as sufficiently large to technically allow placements of further coils, were recorded. ${ }^{21}$

\section{Statistical Methods}

All analyses were performed by the trial statistician (M.C.) according to the published trial protocol. ${ }^{19}$ Analyses were Intent-totreat. The primary outcome was studied by using odds ratios with 95\% confidence intervals (platinum coiling as the reference), and groups were compared by using $\chi^{2}$ tests. Multiple sensitivity analyses were performed for missing values. We performed exploratory stratified analyses, adjusting for rupture status, location, aneurysm size, and the use of adjunct devices. The median packing density was compared between hydrogel and platinum with Mann-Whitney $U$ tests. Packing density is the volume of inserted coils divided by the aneurysm volume. Coil volume (V) was calculated by using the formula $\mathrm{V}=\pi(c / 2)^{2} \mathrm{~L}$, where $\mathrm{c}$ is coil caliber and $\mathrm{L}$ is coil length for coils entered in the procedural case report forms. Aneurysm volumes $\left(\mathrm{V}_{\mathrm{A}}\right)$ were determined by using a simple mathematic model $\mathrm{V}_{\mathrm{A}}=4 / 3 \pi \mathrm{ab}(\mathrm{a}+\mathrm{b}) / 2$, where $\mathrm{a}$ and $\mathrm{b}$ are half the long and short axes of the aneurysm, as entered on the case report forms. The number of deaths was compared with a Fisher exact test. To evaluate the possibility of different results for PRET-1 and PRET-2, we stratified descriptive and safety analyses by groups. All analyses were made with SPSS, Version 23 (IBM, Armonk, New York) by using a significance level of 5\%.

\section{Role of the Funding Source}

The trial was sponsored by the Centre Hospitalier de l'Université de Montréal and funded by MicroVention Terumo. The sponsor and funder had no part in study design, data collection, analysis, or reporting and had no direct or indirect access to the data or source documents. The Steering Committee bears the sole responsibility for all aspects of the trial. 


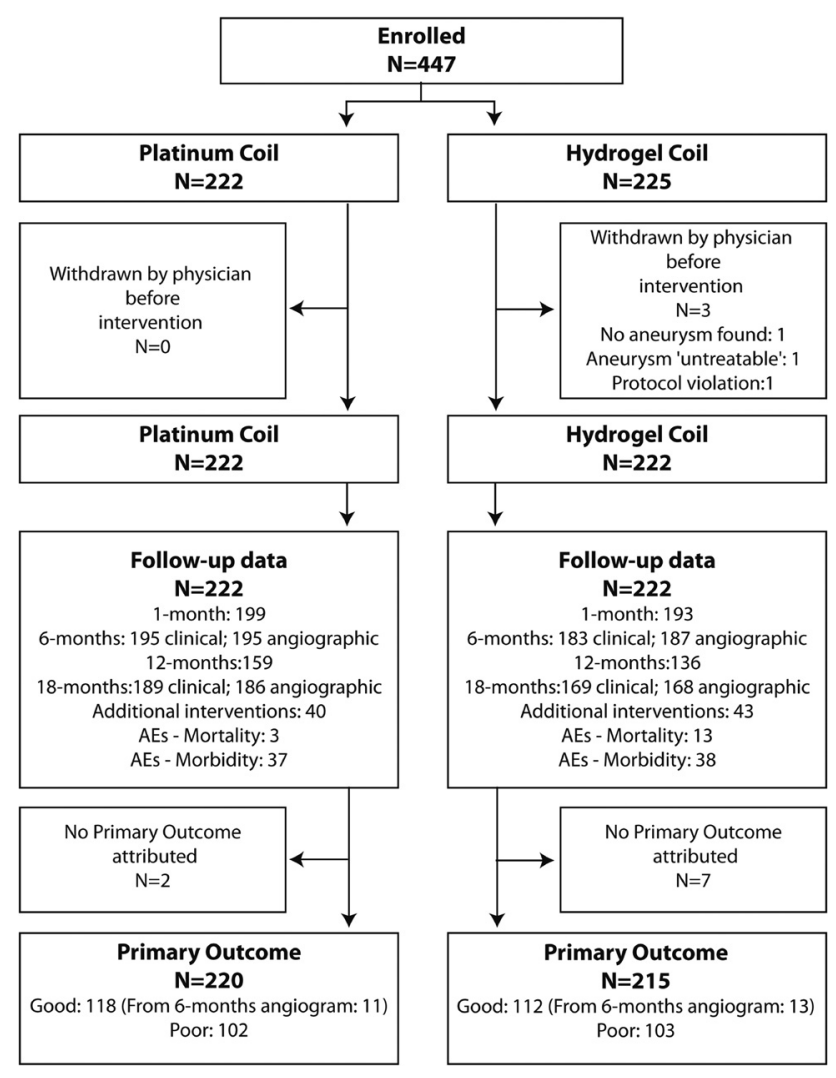

FIG 1. Trial profile. Patients screened are not reported because no eligibility logs were required per protocol. Four hundred forty-seven patients in PRET included 250 in PRET-1 and 197 in PRET- 2 groups.

\section{RESULTS}

Patients were recruited between June 2007 and January 2014. On December 13, 2013, the Steering Committee decided to stop recruitment after the target number of patients $(n=250)$ had been recruited in PRET-1, but before reaching the target number of patients for PRET-2, because the trial was already 2 years behind schedule, recruitment rates were decreasing, and provisions had to be made to cover compensations to participating sites for the 18-month follow-up data. For each group, the number of participants who were randomly assigned, received intended treatment, and were analyzed for the primary outcome is illustrated in the trial profile (Fig 1). The number of screened patients cannot be provided because eligibility logs were not required per protocol. Four hundred forty-seven patients were recruited by 25 centers in 6 countries, 250 in PRET-1 and 197 in PRET-2.

The baseline patient and aneurysm characteristics are shown in Table 1. Groups were comparable.

Three patients $(0.7 \%$; 2 in PRET- 1 and 1 in PRET -2 ; all 3 in the hydrogel group) were withdrawn before any treatment was attempted (1 protocol violation [World Federation of Neurological Societies IV after SAH]), 1 PRET-1 aneurysm judged untreatable, 1 patient in PRET-2 in whom no true recurrence was found). There were 1 crossover from hydrogel to platinum and 1 crossover from platinum to hydrogel, included in the intent-to-treat analyses.

For 9 patients $(2.0 \%$; 2 platinum; 7 hydrogel $)$, the primary outcome could not be attributed because of withdrawn consent (2 hydrogel), lack of follow-up from unrelated mortality (1 hy- drogel), or patients lost to follow-up (2 platinum; 4 hydrogel). Details are provided in On-line Table 1.

For 24 patients $(5.4 \%$; 11 platinum; 13 hydrogel), the 18 month follow-up angiogram was not available and the primary outcome was adjudicated by using the 6-month follow-up angiogram. For 17 patients $(3.8 \%)$, the primary outcome was determined on the basis of a treatment failure and no further angiographic follow-up (no coil deployed: 3 platinum, 5 hydrogel; residual aneurysm: 4 platinum, 5 hydrogel).

The primary outcome, available for $435 / 447$ patients ( $97.3 \%)$, was reached in 102/220 (46.4\%) and 103/215 (47.9\%) patients of the platinum and hydrogel groups, respectively (OR, 1.064; 95\% CI, $0.730-1.550 ; P=.747)$. Each component of the composite primary outcome is detailed for PRET-1 and PRET-2 separately in Table 2. Results for predetermined subgroups (unruptured aneurysms, carotid aneurysms, use of stents, and location) are detailed in On-line Tables 2-4 and in the forest plots (Fig 2).

A poor primary outcome occurred in 55/124 (44.4\%; 95\% CI, $35.5 \%-53.2 \%$ ) patients in PRET-1 with large aneurysms allocated to platinum compared with $63 / 120(52.5 \%$; $95 \%$ CI, $43.4 \%-$ $61.6 \%$ ) patients allocated to hydrogel (OR, 1.387; 95\% CI, $0.838-$ 2.295; $P=.204)$.

In PRET-2 patients who had already presented with a recurrence, a poor primary outcome occurred in $47 / 96$ (49.0\%; 95\% CI, $38.8 \%-59.1 \%)$ and 40/95 (42.1\%; 95\% CI, 32.0\%-52.2\%) patients allocated to platinum and hydrogel, respectively (OR, 0.959; 95\% CI, 0.428-1.342; $P=.342$ ).

The angiographic outcome (residual or recurrent aneurysms) accounted for most primary outcome events (92.2\%); the clinical components (SAH, mass effect, related morbidity or mortality; $n=4$ each) accounted for $16 / 205$ or $7.8 \%$ of primary outcome events. Forty-six patients (10.4\%; 21 platinum, 25 hydrogel) were retreated during the 18 -month follow-up, but 34 other retreatments (7.7\%; 19 platinum, 15 hydrogel) were planned. Follow-up vascular imaging studies were performed by conventional angiography in $75 \%$ and $49 \%$ and by MRA in $25 \%$ and $51 \%$ of patients at 6 and 18 months, respectively.

Four different sensitivity analyses (excluding patients with no angiographic follow-up study from analyses [total $n=426$ ]; counting the 9 patients with no primary outcome as good or poor $[n=444]$; including the 3 patients withdrawn before treatment as treatment failures $[n=447]$ ) did not change the results (On-line Table 5). Repeating all analyses, adjusting for aneurysm location, dimension of the long or short axis, width of the neck, rupture status, or use of stents, did not change the results.

Exploratory analyses, including only patients in whom the target length of hydrogel coils was met (103/120, 85.8\% in PRET-1, and $88 / 95,92.6 \%$ in PRET-2) and repeating all sensitivity analyses, did not show any significant difference between platinum and hydrogel $(P>.08$ in all cases). There was a significant difference between patients in PRET-1 and PRET-2 when patients lost, withdrawn, or without a primary outcome and when the hydrogel target was not met were excluded $(n=395)(P=.025)$ (On-line Table 6).

The median packing density of hydrogel-treated aneurysms (50.9\%) was significantly higher $(P<.001)$ than that in patients 


\begin{tabular}{|c|c|c|c|c|c|c|}
\hline & \multicolumn{2}{|c|}{ PRET-1 } & \multicolumn{2}{|c|}{ PRET-2 } & \multicolumn{2}{|c|}{ PRET } \\
\hline & Platinum & Hydrogel & Platinum & Hydrogel & Platinum & Hydrogel \\
\hline Total No. patients randomized & 125 & 125 & 97 & 100 & 222 & 225 \\
\hline Male sex & $35(28.0 \%)$ & $33(26.4 \%)$ & $33(34 \%)$ & $27(27.0 \%)$ & $68(30.6 \%)$ & $60(26.7 \%)$ \\
\hline Mean age (SD) (yr) & 59 (11) & 58 (11) & $57(12)$ & $56(10)$ & $58(12)$ & 57 (11) \\
\hline Multiple aneurysms & $36(28.8 \%)$ & $36(28.8 \%)$ & $23(23.7 \%)$ & $22(22.0 \%)$ & $59(26.6 \%)$ & $58(25.8 \%)$ \\
\hline \multicolumn{7}{|l|}{ Ruptured aneurysms } \\
\hline No. (\%) of treatment group & $37(29.6 \%)$ & $35(28 \%)$ & $2(2.1 \%)$ & $5(5.0 \%)$ & $39(17.6 \%)$ & $40(17.8 \%)$ \\
\hline No. (\%) WFNS > II & $3(8.1 \%)$ & $8(22.9 \%)$ & 0 & 0 & $3(7.7 \%)$ & $8(20.0 \%)$ \\
\hline \multicolumn{7}{|l|}{ Unruptured aneurysms } \\
\hline mRS at baseline $0-2$ & $85(96.6 \%)$ & $90(100.0 \%)$ & $94(98.9 \%)$ & $94(98.9 \%)$ & $179(97.8 \%)$ & $184(99.5 \%)$ \\
\hline mRS at baseline 3-5 & $3(3.4 \%)$ & 0 & $1(1.1 \%)$ & $1(1.1 \%)$ & $4(2.2 \%)$ & $1(0.5 \%)$ \\
\hline No. (\%) symptomatic & $24(27.3 \%)$ & $18(20 \%)$ & $8(8.4 \%)$ & $9(9.5 \%)$ & $32(17.5 \%)$ & $27(14.6 \%)$ \\
\hline \multicolumn{7}{|l|}{ Aneurysm size (maximal dimension) } \\
\hline Mean (SD) (mm) & $13.3(4.9)$ & $12.7(4.3)$ & $8.6(5.8)$ & $8.3(5.5)$ & $11.3(5.8)$ & $10.7(5.3)$ \\
\hline$\geq 10 \mathrm{~mm}$ & $97(77.6 \%)$ & $96(76.8 \%)$ & $29(29.9 \%)$ & $25(25.0 \%)$ & $126(56.8 \%)$ & $121(53.8 \%)$ \\
\hline $1.0-9.9 \mathrm{~mm}^{\mathrm{a}}$ & $28(22.4 \%)$ & $29(23.2 \%)$ & $68(70.1 \%)$ & $75(75.0 \%)$ & $96(43.2 \%)$ & $104(46.2 \%)$ \\
\hline $10.0-14.9 \mathrm{~mm}$ & $59(47.2 \%)$ & $66(52.8 \%)$ & $16(16.5 \%)$ & $14(14.0 \%)$ & $75(33.8 \%)$ & $80(35.6 \%)$ \\
\hline $15.0-19.9 \mathrm{~mm}$ & $25(20.0 \%)$ & $24(19.2 \%)$ & $5(5.2 \%)$ & $7(7.0 \%)$ & $30(13.5 \%)$ & $31(13.8 \%)$ \\
\hline $20.0-24.9 \mathrm{~mm}$ & $8(6.4 \%)$ & $4(3.2 \%)$ & $6(6.2 \%)$ & $1(1.0 \%)$ & $14(6.3 \%)$ & $5(2.2 \%)$ \\
\hline$\geq 25 \mathrm{~mm}$ & $5(4.0 \%)$ & $2(1.6 \%)$ & $2(2.1 \%)$ & $3(3.0 \%)$ & $7(3.2 \%)$ & $5(2.2 \%)$ \\
\hline \multicolumn{7}{|l|}{ Aneurysm neck } \\
\hline Mean (SD) (mm) & $5.0(2.1)$ & $4.8(1.6)$ & $4.5(2.5)$ & $4.6(2.3)$ & $4.8(2.3)$ & $4.7(1.9)$ \\
\hline Neck $\geq 4.0(\mathrm{~mm})$ & $88(70.4 \%)$ & $94(75.2 \%)$ & $53(54.6 \%)$ & $58(58.0 \%)$ & $141(63.5 \%)$ & $152(67.6 \%)$ \\
\hline Aneurysm location, anterior & $96(76.8 \%)$ & $104(83.2 \%)$ & $69(71.1 \%)$ & 70 (70.0\%) & $165(74.3 \%)$ & $174(77.3 \%)$ \\
\hline Internal carotid & $58(46.4 \%)$ & $69(55.2 \%)$ & $43(44.3 \%)$ & $40(40.0 \%)$ & $101(45.5 \%)$ & $109(48.4 \%)$ \\
\hline Anterior cerebral & $23(18.4 \%)$ & $18(14.4 \%)$ & $21(21.6 \%)$ & $24(24.0 \%)$ & $44(19.8 \%)$ & $42(18.7 \%)$ \\
\hline Middle cerebral & $15(12.0 \%)$ & $17(13.6 \%)$ & $5(5.2 \%)$ & $6(6.0 \%)$ & $20(9.0 \%)$ & $23(10.2 \%)$ \\
\hline Aneurysm location, posterior & $29(23.2 \%)$ & $21(16.8 \%)$ & $28(28.9 \%)$ & $30(30.0 \%)$ & $57(25.7 \%)$ & $51(22.7 \%)$ \\
\hline Basilar & $23(18.4 \%)$ & $13(10.4 \%)$ & $21(21.6 \%)$ & $19(19.0 \%)$ & $44(19.8 \%)$ & $32(14.2 \%)$ \\
\hline Other posterior & $6(4.8 \%)$ & $8(6.4 \%)$ & $7(7.2 \%)$ & $11(11.0 \%)$ & $13(5.9 \%)$ & $19(8.4 \%)$ \\
\hline Intended use of adjunct device & $41(32.8 \%)$ & $42(33.6 \%)$ & $35(36.1 \%)$ & $38(38.0 \%)$ & $76(34.2 \%)$ & $80(35.6 \%)$ \\
\hline
\end{tabular}

Note:-WFNS indicates World Federation of Neurological Societies.

${ }^{a}$ PRET-1 aneurysms recruited as $\geq 10 \mathrm{~mm}$ on noninvasive imaging have been measured using fiducials as $<10 \mathrm{~mm}$ by the core laboratory; median size was $8.6 \mathrm{~mm}$ (mean, $8.3 \pm$ $1.3 \mathrm{~mm})$

Table 2: Primary outcome in all patients in PRET ${ }^{\mathrm{a}}$

\begin{tabular}{|c|c|c|c|c|c|c|}
\hline & \multicolumn{2}{|c|}{ PRET-1 } & \multicolumn{2}{|c|}{ PRET-2 } & \multicolumn{2}{|c|}{ PRET } \\
\hline & $\begin{array}{c}\text { Platinum } \\
(n=124)\end{array}$ & $\begin{array}{c}\text { Hydrogel } \\
(n=120)\end{array}$ & $\begin{array}{l}\text { Platinum } \\
(n=96)\end{array}$ & $\begin{array}{c}\text { Hydrogel } \\
(n=95)\end{array}$ & $\begin{array}{l}\text { Platinum } \\
(n=220)\end{array}$ & $\begin{array}{c}\text { Hydrogel } \\
(n=215)\end{array}$ \\
\hline Primary outcome & $55(44.4 \%)$ & $63(52.5 \%)$ & $47(49.0 \%)$ & $40(42.1 \%)$ & $102(46.4 \%)$ & $103(47.9 \%)$ \\
\hline Major recurrence & $33(26.6 \%)$ & $35(29.2 \%)$ & $33(34.4 \%)$ & $25(26.3 \%)$ & $66(30.0 \%)$ & $60(27.9 \%)$ \\
\hline Retreatment & $11(8.9 \%)$ & $19(15.8 \%)$ & 10 (10.4\%) & $6(6.3 \%)$ & $21(9.5 \%)$ & $25(11.6 \%)$ \\
\hline Initial treatment failure & $3(2.4 \%)$ & $1(0.8 \%)$ & $0(0.0 \%)$ & $4(4.2 \%)$ & $3(1.4 \%)$ & $5(2.3 \%)$ \\
\hline SAH & $1(0.8 \%)$ & $1(0.8 \%)$ & $1(1.0 \%)$ & $1(1.1 \%)$ & $2(0.9 \%)$ & $2(0.9 \%)$ \\
\hline Mass effect & $0(0.0 \%)$ & $1(0.8 \%)$ & $3(3.1 \%)$ & $0(0.0 \%)$ & $3(1.4 \%)$ & $1(0.5 \%)$ \\
\hline Related mortality & $1(0.8 \%)$ & $3(2.5 \%)$ & $0(0.0 \%)$ & $0(0.0 \%)$ & $1(0.5 \%)$ & $3(1.4 \%)$ \\
\hline Related morbidity & $2(1.6 \%)$ & $1(0.8 \%)$ & $0(0.0 \%)$ & $1(1.1 \%)$ & $2(0.9 \%)$ & $2(0.9 \%)$ \\
\hline Last observation carried forward ${ }^{b}$ & $4(3.2 \%)$ & $2(1.7 \%)$ & $0(0.0 \%)$ & $3(3.2 \%)$ & $4(1.8 \%)$ & $5(2.3 \%)$ \\
\hline
\end{tabular}

${ }^{\text {a }}$ Data are numbers.

${ }^{\mathrm{b}}$ Residual aneurysm at initial treatment and no angiographic follow-up.

with platinum coils $(22.0 \%)$. Patients reaching the primary outcome had a significantly lower packing density $(P<.001)$ than patients without a recurrence, whether platinum or hydrogel was used (Table 3).

The periprocedural morbidity, mortality, and adverse events (up to 1 month) have previously been published. ${ }^{20}$ Twenty-seven additional adverse events (14 platinum, 13 hydrogel) occurred after 1 month. Eighteen were serious (7 platinum, 11 hydrogel). Details are provided in Table 4.

Adverse events attributed to inflammation at any time after the procedure (including the first 30 days after treatment) occurred in 6 patients (4 platinum, 2 hydrogel).
Morbidity according to the modified Rankin Scale and mortality are summarized in Table 5. There were 16 deaths (3.6\%, 3 platinum, 13 hydrogel; $P=.011$ ). Two deaths (both hydrogel) related to the initial SAH were reported previously. Two other deaths, one 148 days after the procedure (platinum), the other at an unknown time from an unknown cause following treatment (hydrogel; the patient was lost to follow-up after 1-month) were not initially reported and have been adjudicated to be treatmentrelated delayed deaths. Two deaths 31 and 474 days after treatment were related to SAH at follow-up (hydrogel and platinum, respectively). Two deaths at 64 and 693 days were retreatmentrelated deaths (both hydrogel). Deaths unrelated to the aneurysm 

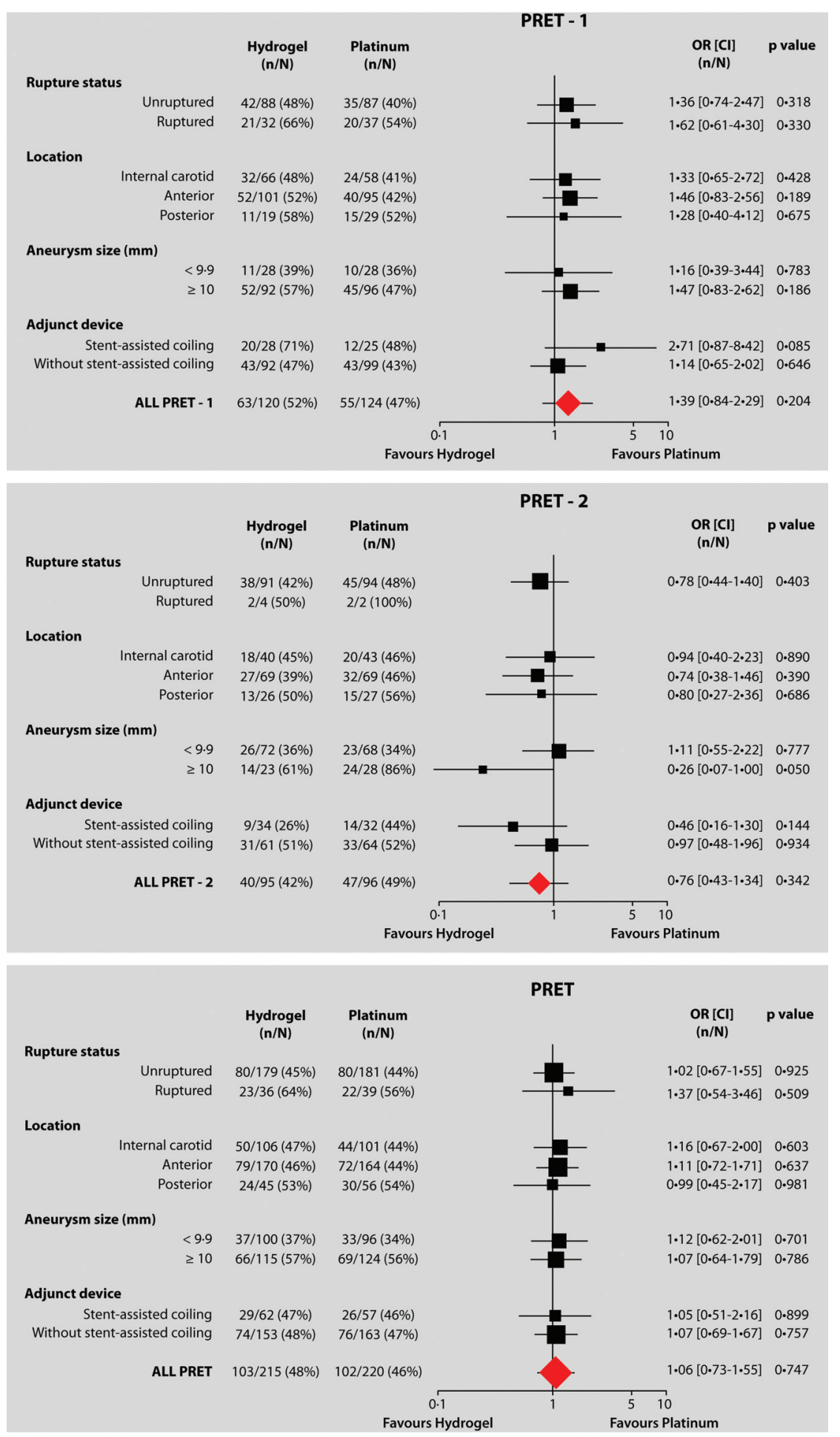

FIG 2. Subgroup analysis of primary outcome in PRET groups.

or its treatment were reported in 8 patients ( 1 platinum; 7 hydrogel). Details are provided in On-line Tables 7 and 8.

\section{DISCUSSION}

A randomized comparison did not show any significant difference between patients in the hydrogel and platinum groups when assessing the primary outcome-a composite of major angiographic recurrence and clinical status. This was true for both pa- tients with large (PRET 1) and recurrent aneurysms (PRET 2) and for each component of the primary outcome. What is not surprising in these high-risk patients, angiographic outcomes were inferior compared with those in other coiling trials. ${ }^{16,17,22,23}$ Safety endpoints were similar for the 2 groups, except for a greater number of deaths unrelated to the aneurysm or treatment (7 versus 1) in the hydrogel group. A careful review of individual cases indicates that this difference is probably a chance finding.

There were more missing primary outcomes in the hydrogel group $(n=7)$ than in the control group $(n=2)$. Although some of these outcomes were missing for reasons unrelated to treatment, reasons are not known for all patients. Sensitivity analyses indicated that missing data would not have affected trial results.

The median packing density of the hydrogel group was significantly higher than in the platinum group, but this finding did not translate into better long-term angiographic results. The volumetric packing density calculation assumes full hydrogel expansion, which might not occur in vivo. Preplanned analyses focusing on patients who have reached the "target hydrogel coiling strategy" did not show better results than those with platinum coiling. There was a correlation between packing density and recurrences for each group studied separately, suggesting that packing density is better understood as an index combining aneurysm characteristics (size, aspect ratio) and technical success of the coiling procedure (with any material) than as an independent mechanistic means to deliberately improve long-term results of embolization, at least with hydrogel in large and recurrent aneurysms.

Residual or recurrent aneurysms are signs that treatment may not be definitive. As expected, there were a few hemorrhagic episodes ( $n=4$ or $0.9 \%$ ) during the 18-month follow-up, but 46 or $10.4 \%$ of patients were retreated and retreatments were planned for 34 other patients.

Inflammatory problems have previously been a concern with the use of hydrogel. ${ }^{14,22,24-27}$ In PRET, adverse events that have been (rightly or wrongly) attributed to inflammation were transient and occurred rarely and with equal frequency in both groups.

Coiling of large and recurrent aneurysms proved safe. ${ }^{20}$ There were few delayed adverse events after 1 month. 
Treatment-related morbidity $(4.0 \%)$ and mortality $(0.7 \%)$ were relatively low for both groups. Unfortunately, the efficacy of coiling remains problematic, with poor long-term primary outcomes in nearly half of patients. Major angiographic recurrence rates were higher than those in other coiling studies, ${ }^{8,28-30}$ but this finding was not unexpected for these high-risk patients; indeed, the trial hypothesized a 50\% recurrence rate for the control group. Similar 50\% recurrence rates for both hydrogel and platinum

Table 3: Packing density versus primary outcome

\begin{tabular}{|c|c|c|c|c|c|c|}
\hline \multirow[b]{2}{*}{ Packing Density ${ }^{a}$} & \multicolumn{2}{|c|}{$\begin{array}{c}\text { Platinum Primary } \\
\text { Outcome } \\
\text { (Angiographic } \\
\text { Outcome Only) }\end{array}$} & \multicolumn{2}{|c|}{$\begin{array}{c}\text { Hydrogel Primary } \\
\text { Outcome } \\
\text { (Angiographic } \\
\text { Outcome Only) }\end{array}$} & \multicolumn{2}{|c|}{$\begin{array}{l}\text { Total Primary } \\
\text { Outcome } \\
\text { (Angiographic } \\
\text { Outcome Only) }\end{array}$} \\
\hline & No & Yes & No & Yes & No & Yes \\
\hline \multicolumn{7}{|l|}{ PRET-1 } \\
\hline Valid No. & 69 & 51 & 57 & 61 & 126 & 112 \\
\hline Percentile 25 & 14.72 & 11.92 & 33.91 & 18.55 & 18.28 & 13.79 \\
\hline Median & 22.18 & 17.13 & 50.92 & 36.93 & 29.60 & 23.47 \\
\hline Median & 35.52 & 27.61 & 89.26 & 58.88 & 53.60 & 46.90 \\
\hline \multicolumn{7}{|l|}{ PRET-2 } \\
\hline Valid No. & 49 & 47 & 55 & 37 & 104 & 84 \\
\hline Percentile 25 & 18.15 & 6.02 & 34.62 & 11.64 & 24.46 & 7.37 \\
\hline Median & 34.05 & 19.66 & 84.80 & 40.38 & 51.49 & 29.12 \\
\hline Percentile 75 & 60.15 & 43.21 & 172.95 & 94.72 & 111.77 & 65.92 \\
\hline \multicolumn{7}{|l|}{ PRET } \\
\hline Valid No. & 118 & 98 & 112 & 98 & 230 & 196 \\
\hline Percentile 25 & 15.23 & 7.65 & 34.27 & 17.91 & 21.81 & 12.55 \\
\hline Median & 24.14 & 17.66 & 62.47 & 38.50 & 37.94 & 25.58 \\
\hline Percentile 75 & 43.72 & 35.71 & 114.34 & 71.66 & 74.87 & 50.58 \\
\hline
\end{tabular}

a Packing density is coil volume $(\mathrm{V})$ divided by aneurysm volume $(\mathrm{VA}) . \mathrm{V}=\pi(\mathrm{c} / 2)^{2} \mathrm{~L}$, where $\mathrm{c}$ is coil caliber and $\mathrm{L}$ is coil length. $V A=4 / 3 \pi a b(a+b) / 2$, where $a$ and $b$ are half the long and short axes of the aneurysm. were reported for the subgroup of patients in the HydrogelCoated Coils versus Bare Platinum Coils for the Endovascular Treatment of Intracranial Aneurysms trial with $>10-\mathrm{mm}$ aneurysms. ${ }^{14}$

Whether patients treated with coiling should be followed to detect recurrences and whether recurrences should be retreated are questions that cannot be answered by the present data. We can only point out that despite retreatment, 3 patients in PRET-2 bled during follow-up; 2 others had progressive mass effect; residual or recurring aneurysms still occurred in $>45 \%$ of patients; and re-retreatments were associated with serious adverse events in 3 patients.

How patients with large or recurrent aneurysms should be managed remains an open question. In the landmark International Subarachnoid Aneurysm Trial (ISAT), which has established the coiling of ruptured aneurysms compared with clipping, clinical outcomes of patients with aneurysms of $>10 \mathrm{~mm}$ $(n=155)$ were similar (relative risk, 0.96; 95\% CI, 0.65-1.42). Thus, clipping, reputed but not proved, at least for unruptured aneurysms, ${ }^{31}$ to be more durable than coiling, is a reasonable option for some of these patients, though the long-term follow-up ISAT data con-

Table 4: Adverse events ${ }^{a}$

\begin{tabular}{|c|c|c|c|c|c|c|}
\hline & \multicolumn{2}{|c|}{ PRET-1 } & \multicolumn{2}{|c|}{ PRET-2 } & \multicolumn{2}{|c|}{ PRET } \\
\hline & $\begin{array}{l}\text { Platinum } \\
(n=125)\end{array}$ & $\begin{array}{c}\text { Hydrogel } \\
(n=123)\end{array}$ & $\begin{array}{l}\text { Platinum } \\
(n=97)\end{array}$ & $\begin{array}{c}\text { Hydrogel } \\
(n=99)\end{array}$ & $\begin{array}{l}\text { Platinum } \\
(n=222)\end{array}$ & $\begin{array}{l}\text { Hydroge } \\
(n=222)\end{array}$ \\
\hline Total & 24 (19.2\%) & $26(21.1 \%)$ & $13(13.4 \%)$ & 15 (15.2\%) & 37 (16.7\%) & $41(18.5 \%)$ \\
\hline SAH during follow-up & $1(0.8 \%)$ & $0(0.0 \%)$ & $1(1.0 \%)$ & $2(2.0 \%)$ & $2(0.9 \%)$ & $2(0.9 \%)$ \\
\hline Mass effect & $0(0.0 \%)$ & $1(0.8 \%)$ & $2(2.1 \%)$ & $0(0.0 \%)$ & $2(0.9 \%)$ & $1(0.5 \%)$ \\
\hline Stroke & $1(0.8 \%)$ & $2(1.6 \%)$ & $1(1.0 \%)$ & $0(0.0 \%)$ & $2(0.9 \%)$ & $2(0.9 \%)$ \\
\hline TIA & $0(0.0 \%)$ & $0(0.0 \%)$ & $0(0.0 \%)$ & $1(1.0 \%)$ & $0(0.0 \%)$ & $1(0.5 \%)$ \\
\hline Inflammatory & $2(1.6 \%)$ & $0(0.0 \%)$ & $0(0.0 \%)$ & $0(0.0 \%)$ & $2(0.9 \%)$ & $0(0.0 \%)$ \\
\hline Subdural hematoma & $0(0.0 \%)$ & $0(0.0 \%)$ & $0(0.0 \%)$ & $1(1.0 \%)$ & $0(0.0 \%)$ & $1(0.5 \%)$ \\
\hline Non-neurologic & $3(2.4 \%)$ & $1(0.8 \%)$ & $0(0.0 \%)$ & $0(0.0 \%)$ & $3(1.4 \%)$ & $1(0.5 \%)$ \\
\hline Retreatment-related & $1(0.8 \%)$ & $4(3.3 \%)$ & $2(2.1 \%)$ & $1(1.0 \%)$ & $3(1.4 \%)$ & $5(2.3 \%)$ \\
\hline Periprocedural event & $16(12.8 \%)$ & $18(14.6 \%)$ & $7(7.2 \%)$ & $10(10.1 \%)$ & $23(10.4 \%)$ & $28(12.6 \%)$ \\
\hline Serious & 15 (12.0\%) & 15 (12.2\%) & $5(5.2 \%)$ & $10(10.1 \%)$ & $20(9.0 \%)$ & 25 (11.3\%) \\
\hline Delayed & $5(4.0 \%)$ & $7(5.7 \%)$ & $2(2.1 \%)$ & $4(4.0 \%)$ & $7(3.2 \%)$ & $11(5.0 \%)$ \\
\hline Periprocedure & $10(8.0 \%)$ & $8(6.5 \%)$ & $3(3.1 \%)$ & $6(6.1 \%)$ & $13(5.9 \%)$ & $14(6.3 \%)$ \\
\hline
\end{tabular}

${ }^{a}$ Adverse events were recorded during the trial. Periprocedural events (within 1 month of procedure) are lumped together. Data are numbers.

Table 5: Morbidity and mortality according to mRS at last follow-up ${ }^{a}$

\begin{tabular}{|c|c|c|c|c|c|c|}
\hline \multirow[b]{2}{*}{$\mathrm{mRS}$} & \multicolumn{2}{|c|}{ PRET-1 } & \multicolumn{2}{|c|}{ PRET-2 } & \multicolumn{2}{|c|}{ PRET } \\
\hline & $\begin{array}{l}\text { Platinum } \\
(n=125)\end{array}$ & $\begin{array}{l}\text { Hydrogel } \\
(n=123)\end{array}$ & $\begin{array}{l}\text { Platinum } \\
(n=97)\end{array}$ & $\begin{array}{c}\text { Hydrogel } \\
(n=99)\end{array}$ & $\begin{array}{l}\text { Platinum } \\
(n=222)\end{array}$ & $\begin{array}{c}\text { Hydrogel } \\
(n=222)\end{array}$ \\
\hline 0 & 88 (70.4\%) & 77 (62.6\%) & $60(61.9 \%)$ & $67(67.7 \%)$ & 148 (66.7\%) & 144 (64.9\%) \\
\hline 1 & 19 (15.2\%) & $25(20.3 \%)$ & 23 (23.7\%) & $16(16.2 \%)$ & 42 (18.9\%) & $41(18.5 \%)$ \\
\hline 2 & $7(5.6 \%)$ & $7(5.7 \%)$ & $10(10.3 \%)$ & $9(9.1 \%)$ & $17(7.7 \%)$ & $16(7.2 \%)$ \\
\hline 3 & $1(0.8 \%)$ & $3(2.4 \%)$ & $3(3.1 \%)$ & $0(0.0 \%)$ & $4(1.8 \%)$ & $3(1.4 \%)$ \\
\hline 4 & $5(4.0 \%)$ & $1(0.8 \%)$ & $1(1.0 \%)$ & $3(3.0 \%)$ & $6(2.7 \%)$ & $4(1.8 \%)$ \\
\hline 5 & $2(1.6 \%)$ & $0(0.0 \%)$ & $0(0.0 \%)$ & $1(1.0 \%)$ & $2(0.9 \%)$ & $1(0.5 \%)$ \\
\hline 6 & $3(2.4 \%)$ & $10(8.1 \%)$ & $0(0.0 \%)$ & $3(3.0 \%)$ & $3(1.4 \%)$ & $13(5.9 \%)$ \\
\hline
\end{tabular}

${ }^{a}$ Data are numbers. 
firm a low rebleed risk after de novo coiling and long-term outcome superiority over clipping. ${ }^{9}$ Patients with large ruptured aneurysms could be offered participation in the ISAT II trial. ${ }^{32}$ There are no randomized data for unruptured aneurysms, but a trial is ongoing. ${ }^{4}$ Although meta-analyses of case series have reported improved angiographic results with stent-assisted coiling $^{12}$ or flow diversion ${ }^{10}$ compared with historical coiling controls, preliminary results from the Flow Diversion in Intracranial Aneurysm Treatment trial have so far been below expectations. ${ }^{11}$ Participation in these or other ongoing trials may be the best way to manage these high-risk patients. ${ }^{33-36}$

The PRET trial had several limitations. Operators could not be blinded to coil type. This may have affected case selection and coil selection and perhaps may have even modified the extent and completeness of the coiling procedure. Aneurysm volumes were not directly measured but were extrapolated from aneurysm dimensions; this method typically overestimates volumes and underestimates packing density. This is unlikely to have biased the comparison between groups. Recruitment was slowing down in the last years of enrollment, perhaps because treatment alternatives, such as flow diverters, were increasingly used for large and recurrent aneurysms. This may not affect the generalizability of conclusions, equally disappointing for both groups. The PRET-2 substudy was interrupted before the target number of patients was enrolled. Given the similarities in overall trial results, it is unlikely that a convincing difference between groups would have been shown had the trial reached its target. The relatively short 18month follow-up was not completed by all patients, and the primary endpoint had to be imputed from the 6-month follow-up angiogram in $5.5 \%$ of patients. Short follow-up periods may not have captured all clinical consequences of recurrences, such as retreatments, along with the associated morbidity. Data monitoring was done on-line, with no local site visits to verify the data. Brain imaging studies were not imposed by protocol to verify the absence of asymptomatic complications. These perceived deficiencies are expected to affect treatment groups in a balanced manner.

\section{ACKNOWLEDGMENTS}

First and foremost, we acknowledge the support of patients and their relatives who agreed to participate in the study and who provided us with follow-up data. We also thank the medical, radiologic, and nursing staff of all participating centers. We are grateful to members of the Data Safety and Monitoring Committees (Sylvain Lanthier, Allan Fox). Implementing a study such as PRET requires considerable effort for a long time. Special thanks are due to Guylaine Gevry and her team at the PRET Coordination Head Office in Montreal for their outstanding work and indefectible commitment.

\section{PRET Trial Collaborators}

The PRET trial collaborators are listed in the order that participating sites joined the trial, with the number of patients recruited given in parentheses. Centre Hospitalier de l'Université de Montréal-Notre Dame Hospital, Montreal, Quebec, Canada: Principal Investigators, Jean Raymond, Alain Weill, and Daniel Roy; Coordinator, Ruby Klink (120). The Methodist Hospital, Hous- ton, Texas: Principal Investigators, Richard Klucznik and Orlando Diaz; Coordinator, Marilyn Bautista (12). Kobe City Medical Center General Hospital, Kobe, Japan: Principal Investigators, Nobuyuki Sakai and Horotoshi Imamura (4). Medical University of South Carolina, Charleston, South Carolina: Principal Investigators, Aquilla Turk and Raymond Turner; Coordinator, Adrian Parker (26). State University of New York at Stony Brook University Medical Center, Stony Brook, New York: Principal Investigator, Henry Woo; Coordinators, Susan Fiore and Dawn Madigan (20). Oregon Health and Science University, Portland, Oregon: Principal Investigator, Stanley Barnwell; Coordinator, Sarah Ross-Jamieson (63). Cleveland Clinic, Cleveland, Ohio: Principal Investigator, Thomas Masaryk; Coordinator, Terese Wheeler (1). Centre Hospitalier Universitaire de Nancy-Hôpital Central, Nancy, France: Principal Investigator, Serge Bracard; special thanks to Dr Anne Laure Derelle (8). Leeds General Infirmary, Leeds, United Kingdom: Principal Investigator, Tony Goddard; Coordinator, Jonathan Pearce (also, central coordinator for the United Kingdom) (10). The Ottawa Hospital, Ottawa, Ontario, Canada: Principal Investigator, Marlise Santos; Coordinator, Betty Anne Schwarz (14). Instituto de Neurocirugía Dr Asenjo, Santiago, Chile: Principal Investigators, Juan-Gabriel Sordo Jara and Eduardo Bravo (14). University of Florida (Shands Hospital), Gainesville, Florida: Principal Investigators, J. Mocco and Brian Hoh; Coordinators, Bree Burks and Nicolle Wilson-Davis (29). University of Virginia Health System, Charlottesville, Virginia: Principal Investigator, Avery Evans; Coordinators, Claire McKinley and Thomas Tandy (39). Centre Hospitalier Universitaire de Nantes-Hôpital Guillaume et René Laennec, Nantes, France: Principal Investigator, Hubert Desal (23). West Virginia University Hospital, Morgantown, West Virginia: Principal Investigator, Jeffrey Carpenter; Coordinator, Jennifer Domico (8). State University of New York Upstate Medical University, Syracuse, New York: Principal Investigator, Eric Deshaies; Coordinators, Tina Craig, Kim Kasprowicz, Susan Hemingway, and Mark Villwock (10). University of Cincinnati Medical Center, Cincinnati, Ohio: Principal Investigator, Andrew Ringer; Coordinator, Rebecca Reinert (4). Washington University in St. Louis, St. Louis, Missouri: Principal Investigator, Christopher Moran; Coordinator, Angela Campbell (6). Queens Medical Centre, Nottingham, United Kingdom: Principal Investigator, Robert Lenthall; Coordinator, Alison Southam (12). Saint Francis Medical Center, Cape Girardeau, Missouri: Principal Investigator, Louis Caragine; Coordinators, Adrienne Jones and Kathy O'Howell (2). University of Oklahoma Health Sciences Center, Oklahoma City, Oklahoma: Principal Investigator, Steven Hoover; Coordinators, Brian Bridges and Bradley Hightower (11). Centre Hospitalier Sainte Anne, Paris, France: Principal Investigator, Olivier Naggara (2). University of Mississippi Health Care, Jackson, Mississippi: Principal Investigator, Razvan Buciuc; Coordinator, David Gordy (3). University of Alberta Hospital, Edmonton, Alberta, Canada: Principal Investigator, Tim E. Darsaut (2). Vanderbilt University Medical Center, Nashville, Tennessee: Principal Investigator, J. Mocco; Coordinators, Chesney Sarah Oravec and Jessica Sparks Marlin (4). Angiographic Core Laboratory: Phil White, Robin Sellar, Lynn Forrester, Newcastle and Edinburgh, UK. 
Disclosures: Jean Raymond-RELATED: Grant: MicroVention, Comments: unrestricted investigator-led research grant*; UNRELATED: Grants/Grants Pending: Canadian Institutes of Health Research and Johnson \& Johnson, DePuy Synthes (Codman Neuro), Comments: peer-review grant for Canadian UnRuptured Endovascular versus Surgery study and unrestricted investigator-led research grant for the Delta study, respectively. * Avery J. Evans—RELATED: Grant: MicroVention.* J. MoccoRELATED: Grant: Stryker, Penumbra, Medtronic, Comments: research funding*; UNRELATED: Consultancy: TSP, Cerebrotech Medical, Rebound Medical, Pulsar Vascular, EndoStream; Stock/Stock Options: Blockade Medical, TSP, Cerebrotech Medical. Aquilla S. Turk—RELATED: Grant: Stryker, MicroVention, Penumbra, Medina Medical, Covidien, Comments: research funding*; Consulting Fee or Honorarium: Stryker, MicroVention, Penumbra, Medina Medical, Comments: honorarium, consulting; Support for Travel to Meetings for the Study or Other Purposes: Stryker, MicroVention, Penumbra, Medina Medical, Comments: coverage for airfare or hotel; UNRELATED: Consultancy: Stryker, MicroVention, Penumbra, Medina Medical; Grants/Grants Pending: Stryker, MicroVention, Penumbra, Medina Medical*; Payment for Lectures Including Service on Speakers Bureaus: Stryker, MicroVention, Penumbra, Medina Medical; Stock/Stock Options: Medina Medical, Three Rivers. Raymond D. Turner—RELATED: Grant: MicroVention, Penumbra, Medtronic, Pulsar Vascular, Sequent Medical, Stryker*; Consulting Fee or Honorarium: MicroVention, Penumbra, Medtronic, Pulsar Vascular, Sequent Medical, Stryker, Blockade Medical, Q'Apel Medical, Rebound Medical, Codman Neuro. David Fiorella—RELATED: Grant: Sequent Medical, MicroVention, National Institutes of Health, Comments: Principal Investigator responsibilities for trials, ${ }^{*}$ funding indirectly related to study at hand; Consulting Fee or Honorarium: Sequent Medical, MicroVention, Medtronic, Stryker, Penumbra, Comments: proctoring and consulting; Support for Travel to Meetings for the Study or Other Purposes: Sequent Medical, MicroVention, Medtronic, Stryker, Penumbra; UNRELATED: Consultancy: Sequent Medical, MicroVention, Medtronic, Stryker, Penumbra, Codman Neuro/JNJ; Payment for Lectures Including Service on Speakers Bureaus: MicroVention, Penumbra; Patents (Planned, Pending or (ssued): Codman Neuro/Johnson \& Johnson. * Alain Weill—UNRELATED: Consultancy: Balt Extrusion, Comments: member of the Adverse Event Committee of LEO 2 study, a registry of LEO stent used in France.* Philip M. White-UNRELATED: Consultancy: MicroVention, Codman Neuro, Comments: for educational training; Grants/Grants Pending: MicroVention Terumo for STABILISE thrombectomy trial*; Travel/Accommodations/Meeting Expenses Unrelated to Activities Listed: MicroVention Terumo. Daniel Roy—RELATED: Grant: MicroVention, Comments: unrestricted research fund for PRET.* *Money paid to the institution.

\section{REFERENCES}

1. Guglielmi G, Viñuela F, Dion J, et al. Electrothrombosis of saccular aneurysms via endovascular approach, part 2: preliminary clinical experience. J Neurosurg 1991;75:8-14 CrossRef Medline

2. Guglielmi G, Viñuela F, Sepetka I, et al. Electrothrombosis of saccular aneurysms via endovascular approach, part 1: electrochemical basis, technique, and experimental results. J Neurosurg 1991;75:1-7 CrossRef Medline

3. Molyneux A, Kerr R, Stratton I, et al; International Subarachnoid Aneurysm Trial (ISAT) Collaborative Group. International Subarachnoid Aneurysm Trial (ISAT) of neurosurgical clipping versus endovascular coiling in $\mathbf{2 1 4 3}$ patients with ruptured intracranial aneurysms: a randomised trial. Lancet 2002;360:1267-74 CrossRef Medline

4. Darsaut TE, Findlay JM, Raymond J. The design of the Canadian UnRuptured Endovascular versus Surgery (CURES) trial. Can J Neurol Sci 2011;38:236-41 CrossRef Medline

5. Raymond J, Molyneux AJ, Fox AJ, et al; TEAM Collaborative Group. The TEAM trial: safety and efficacy of endovascular treatment of unruptured intracranial aneurysms in the prevention of aneurysmal hemorrhages: a randomized comparison with indefinite deferral of treatment in $\mathbf{2 0 0 2}$ patients followed for $\mathbf{1 0}$ years. Trials 2008; 9:43 CrossRef Medline

6. Crobeddu E, Lanzino G, Kallmes DF, et al. Review of 2 decades of aneurysm-recurrence literature, part 2: managing recurrence after endovascular coiling. AJNR Am J Neuroradiol 2013;34:481-85 CrossRef Medline

7. Naggara ON, Lecler A, Oppenheim C, et al. Endovascular treatment of intracranial unruptured aneurysms: a systematic review of the literature on safety with emphasis on subgroup analyses. Radiology 2012;263:828-35 CrossRef Medline

8. Raymond J, Guilbert F, Weill A, et al. Long-term angiographic re- currences after selective endovascular treatment of aneurysms with detachable coils. Stroke 2003;34:1398-403 CrossRef Medline

9. Molyneux AJ, Birks J, Clarke A, et al. The durability of endovascular coiling versus neurosurgical clipping of ruptured cerebral aneurysms: 18 year follow-up of the UK cohort of the International Subarachnoid Aneurysm Trial (ISAT). Lancet 2015;385:691-97 CrossRef Medline

10. Brinjikji W, Murad MH, Lanzino G, et al. Endovascular treatment of intracranial aneurysms with flow diverters: a meta-analysis. Stroke 2013;44:442-47 CrossRef Medline

11. Raymond J, Gentric JC, Darsaut TE, et al. Flow diversion in the treatment of aneurysms: a randomized care trial and registry. J Neurosurg 2016 Nov 4:1-9. [Epub ahead of print] Medline

12. Hong $Y$, Wang YJ, Deng Z, et al. Stent-assisted coiling versus coiling in treatment of intracranial aneurysm: a systematic review and meta-analysis. PLoS One 2014;9:e82311 CrossRef Medline

13. Phan K, Huo YR, Jia F, et al. Meta-analysis of stent-assisted coiling versus coiling-only for the treatment of intracranial aneurysms. J Clin Neurosci 2016;31:15-22 CrossRef Medline

14. White PM, Lewis SC, Gholkar A, et al; HELPS trial collaborators. Hydrogel-coated coils versus bare platinum coils for the endovascular treatment of intracranial aneurysms (HELPS): a randomised controlled trial. Lancet 2011;377:1655-62 CrossRef Medline

15. Broeders JA, Ahmed Ali U, Molyneux AJ, et al. Bioactive versus bare platinum coils for the endovascular treatment of intracranial aneurysms: systematic review and meta-analysis of randomized clinical trials. J Neurointerv Surg 2016;8:898-908 CrossRef Medline

16. McDougall CG, Johnston SC, Gholkar A, et al; MAPS Investigators. Bioactive versus bare platinum coils in the treatment of intracranial aneurysms: the MAPS (Matrix and Platinum Science) trial. AJNR Am J Neuroradiol 2014;35:935-42 CrossRef Medline

17. Coley S, Sneade M, Clarke A, et al. Cerecyte coil trial: procedural safety and clinical outcomes in patients with ruptured and unruptured intracranial aneurysms. AJNR Am J Neuroradiol 2012;33: 474-80 CrossRef Medline

18. Molyneux AJ, Clarke A, Sneade M, et al. Cerecyte coil trial: angiographic outcomes of a prospective randomized trial comparing endovascular coiling of cerebral aneurysms with either Cerecyte or bare platinum coils. Stroke 2012;43:2544-50 CrossRef Medline

19. Raymond J, Roy D, White PM, et al; Icone Collaborative Group. A randomized trial comparing platinum and hydrogel-coated coils in Patients Prone to Recurrence after Endovascular Treatment (the PRET trial). Interv Neuroradiol 2008;14:73-83 Medline

20. Raymond J, Klink R, Chagnon M, et al; PRET Collaborative Group. Patients prone to recurrence after endovascular treatment: periprocedural results of the PRET randomized trial on large and recurrent aneurysms. AJNR Am J Neuroradiol 2014;35:1667-76 CrossRef Medline

21. Roy D, Milot G, Raymond J. Endovascular treatment of unruptured aneurysms. Stroke 2001;32:1998-2004 CrossRef Medline

22. White PM, Lewis SC, Nahser H, et al; HELPS Trial Collaboration. HydroCoil Endovascular Aneurysm Occlusion and Packing Study (HELPS trial): procedural safety and operator-assessed efficacy results. AJNR Am J Neuroradiol 2008;29:217-23 CrossRef Medline

23. Molyneux AJ, Kerr RS, Yu LM, et al; International Subarachnoid Aneurysm Trial (ISAT) Collaborative Group. International subarachnoid aneurysm trial (ISAT) of neurosurgical clipping versus endovascular coiling in 2143 patients with ruptured intracranial aneurysms: a randomised comparison of effects on survival, dependency, seizures, rebleeding, subgroups, and aneurysm occlusion. Lancet 2005;366:809-17 CrossRef Medline

24. Im SH, Han MH, Kwon BJ, et al. Aseptic meningitis after embolization of cerebral aneurysms using hydrogel-coated coils: report of three cases. AJNR Am J Neuroradiol 2007;28:511-12 Medline

25. Meyers PM, Lavine SD, Fitzsimmons BF, et al. Chemical meningitis after cerebral aneurysm treatment using two second-generation aneurysm coils: report of two cases. Neurosurgery 2004;55:1222 CrossRef Medline

26. Turner RD, Byrne JV, Kelly ME, et al. Delayed visual deficits and monocular blindness after endovascular treatment of large and gi- 
ant paraophthalmic aneurysms. Neurosurgery 2008;63:469-74; discussion 474-75 CrossRef Medline

27. Xu DS, Hurley MC, Batjer HH, et al. Delayed cranial nerve palsy after coiling of carotid cavernous sinus aneurysms: case report. Neurosurgery 2010;66:E1215-16 CrossRef Medline

28. Byrne JV, Sohn MJ, Molyneux AJ, et al. Five-year experience in using coil embolization for ruptured intracranial aneurysms: outcomes and incidence of late rebleeding. J Neurosurg 1999;90:656-63 CrossRef Medline

29. Cognard C, Weill A, Castaings L, et al. Intracranial berry aneurysms: angiographic and clinical results after endovascular treatment. $R a$ diology 1998;206:499-510 CrossRef Medline

30. Adams WM, Laitt RD. Aneurysm remnants. Neurointerventionist 2000;1:74-83

31. Kotowski M, Naggara O, Darsaut TE, et al. Safety and occlusion rates of surgical treatment of unruptured intracranial aneurysms: a systematic review and meta-analysis of the literature from 1990 to 2011. J Neurol Neurosurg Psychiatry 2013;84:42-48 CrossRef Medline
32. Darsaut TE, Jack AS, Kerr RS, et al. International Subarachnoid Aneurysm Trial-ISAT, part II: study protocol for a randomized controlled trial. Trials 2013;14:156 CrossRef Medline

33. Darsaut TE, Raymond J. The design of the STenting in Aneurysm Treatments (STAT) trial. J Neurointerv Surg 2012;4:178-81 CrossRef Medline

34. Raymond J, Darsaut TE, Guilbert F, et al. Flow diversion in aneurysms trial: the design of the FIAT study. Interv Neuroradiol 2011;17: 147-53 Medline Medline

35. Turk AS 3rd, Martin RH, Fiorella D, et al. Flow diversion versus traditional endovascular coiling therapy: design of the prospective LARGE aneurysm randomized trial. AJNR Am J Neuroradiol 2014; 35:1341-45 CrossRef Medline

36. Gory B, Bonafé A, Pierot L, et al. Safety and efficacy of flow-diverter stents in endovascular treatment of intracranial aneurysm: interest of the prospective DIVERSION observational study. J Neuroradiol 2014;41:93-96 CrossRef Medline 